\title{
Effect of Curing Time and Temperature on the Structural Stability of Melamine Formaldehyde Polymers
}

\author{
Phisan Katekomol \\ Faculty of Science, Department of Chemistry, Naresuan University, Tha-Pho, Phitsanulok, \\ Thailand, 65000 \\ phisank@nu.ac.th
}

Keywords: melamine-formaldehyde, melamine resins, curing temperature, curing time

\begin{abstract}
Melamine-formaldehyde polymer was synthesized into the form of self-supporting films. The structural stability of these films was investigated by means of spectroscopy and thermal analysis. The curing temperature and time has effects on the structural stability of the resulting polymer films. In this work, polymer film cured at $200 \mathrm{oC}$ for 12 hours showed highest structural stability compared to those cured at lower temperature and shorter time duration.
\end{abstract}

\section{Introduction}

Melamine-formaldehyde (MF) polymers have been synthesized and applied as various coating and adhesive materials. [1,2,3] Since melamine is produced in industrial scale annually and can be cross-linked by reacting with formaldehyde in acidic or basic conditions, it is a promising cheap starting material for synthesizing polymer networks with various applications. [4] Polymer networks with triazine functionalities have been proven to be successful as being both support for metallic catalysts and as metal-free catalysts.[5,6] This fact points to the various potential applications of polymer networks based on melamine resins. Structural design and modification of such polymer networks is also important in order to reach their maximum application potentials. Employing softtemplating technique, hexamethoxymethyl melamine (HMMM), a pre-alkylated melamineformaldehyde resin has been successfully demonstrated to be a good starting material for the synthesis of mesoporous polymer networks with adjustable nanostructures.[7] Curing process is an important process in the synthesis of MF polymers. After condensation polymerization of methyloated melamine, further condensation and structural rearrangements can occur during the curing process.[7] There has been a study done extensively on the curing process of such polymers in order to study its mechanisms using trimethylol melamine. It has been shown that the curing process of such material is a two-stage process and occurs above $140{ }^{\circ} \mathrm{C}$.[8] In this article a small additional experiment is conducted to show the effect of curing temperature and time on the structural stability of the already-cured resulting MF polymers.

\section{Experimental Procedure}

Materials. Melamine 99.0\% (Merck), 37\% formaldehyde solution (Loba chemie), 99.9\% Methanol (RCI Labscan), 98\% NaOH pellets (Sigma-Aldrich) and 37\% Hydrochloric acid (LabScan Analytical Sciences) were used as received.

Synthesis of MF polymer films. The synthesis of MF polymer films was first done by methylolation of melamine by a method mentioned in an earlier work.[9] In this work, the $\mathrm{pH}$ of $20 \mathrm{ml}$ of $37 \%$ formaldehyde solution was adjusted to 8 by $10 \% \mathrm{NaOH}$ solution. $4.7 \mathrm{~g}$ of melamine is then added to the solution, the mixture is heated to $75{ }^{\circ} \mathrm{C}$ with continuous stirring. When the solution became clear, the reaction is stopped and the mixture was immediately cooled down to the temperature of liquid carbon dioxide. The resulting white gel was then picked out and put in an open container such as a Petri dish and left to dry at $50{ }^{\circ} \mathrm{C}$ in a vacuum oven overnight. The resulting trimethyololmelamine (TMM) appeared as lumps of white solid which was then ground to fine powder for further characterizations. The synthesis of MF polymer films was done similarly to 
a method described earlier for the cross-linking of hexamethoxymethylmelamine (HMMM).[7] The cross-linking of TMM was done in methanol under acidic condition. $1 \mathrm{~g}$ of TMM powder was added to $50 \mathrm{~mL}$ methanol along with $0.1 \mathrm{ml} \mathrm{HCl}$. The mixture was then heated and stirred at $100{ }^{\circ} \mathrm{C}$ under reflux for 2 hours. The resulting clear solution was then poured into a Petri dish; excess methanol was left to evaporate at room temperature. The resulting material was a transparent gel coated on the Petri dish which was later cured in air oven at different temperatures and lengths of time.

Curing Experiment. MF polymer films from cross-linked TMM were cured in air oven at different temperatures and lengths of time. The curing temperatures are 100,150 and $200{ }^{\circ} \mathrm{C}$ and the curing time was set to 1 and 12 hours for each temperature. After complete methanol evaporation, the MF gel was immediately subjected to a set temperature without any ramping. The drastic difference in the curing time was designed to intensify any possible influence of this parameter on the structural stability of the resulting MF polymer films. The resulting films were then ground to find powder for further characterizations with infrared spectroscopy and thermal analysis.

Characterization Methods. Nuclear magnetic resonance. ${ }^{1} \mathrm{H}-\mathrm{NMR}$ was carried out using a Bruker Advanced 400. The sample was dissolved in $\mathrm{D}_{2} \mathrm{O}$ and $1 \mathrm{D}$ chemical shifts were recorded at room temperature.

Fourier transform infrared spectroscopy. FTIR studies were carried out in ATR mode using a Perkin Elmer Spectrum GX. The spectra were recorded in the wavenumber range of $4000-400 \mathrm{~cm}^{-1}$ at $4 \mathrm{~cm}^{-1}$ resolution.

Thermal analysis. Thermogravimetric analysis (TGA) and differential scanning calorimetry (DSC) were carried out using a Mettler Toledo STAR $^{\mathrm{e}}$ thermal analyzer. The samples were gradually heated up from 50 to $700{ }^{\circ} \mathrm{C}$ under normal atmosphere with the heating rate of $5{ }^{\circ} \mathrm{C} \min ^{-1}$. For TGA, the initial weight of samples ranged between $8-10 \mathrm{mg}$.

\section{Result and Discussion}

The resulting TMM was obtained, after grounding, in the form of white powder. The powder was water soluble which was a characteristic property of TMM already described in the original paper [9]. $10 \mathrm{mg}$ of the powder was dissolved in D2O for $1 \mathrm{H}-\mathrm{NMR}$ measurement which yielded a pronounced signal at $4.8 \mathrm{ppm}$ (Fig. 1), a characteristic signal of methylene protons which are the dominant type of protons in this molecule. The signal was further confirmed by comparison with a simulated signal obtained from www.nmrdb.org to be that of the methylene protons of TMM. 


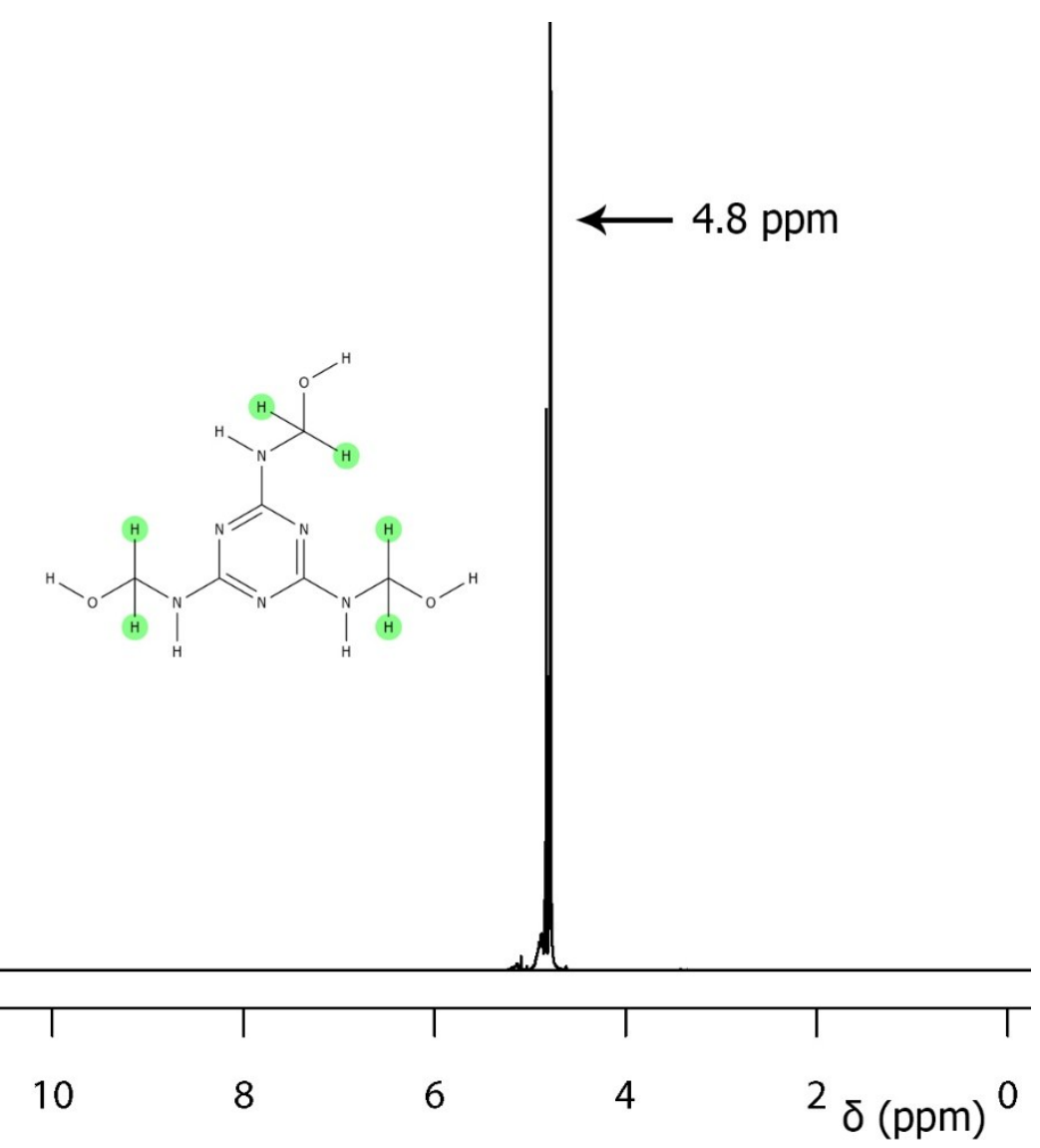

Fig. $1{ }^{1} \mathrm{H}-\mathrm{NMR}$ signal of TMM with $\mathrm{D}_{2} \mathrm{O}$ as solvent showing the pronounced methylene protons chemical shift at $4.8 \mathrm{ppm}$

The FTIR spectra of TMM and uncured, partially cross-linked polymer in the gel state were compared in Fig 2. The spectrum of TMM shows obvious vibrations of secondary amine at 3320 and $3716 \mathrm{~cm}^{-1}$. Ring vibration of $\mathrm{C}=\mathrm{N}$ can also be observed at $1515 \mathrm{~cm}^{-1}$. The characteristic methylol C-H vibration is observed at $2955 \mathrm{~cm}^{-1}$. As for the uncured gel, similar vibration bands can be observed with less pronounced characteristic which suggests the formation of a polymer network. However, the characteristic methylol stretching band is still visible which suggests the incomplete condensation reaction.

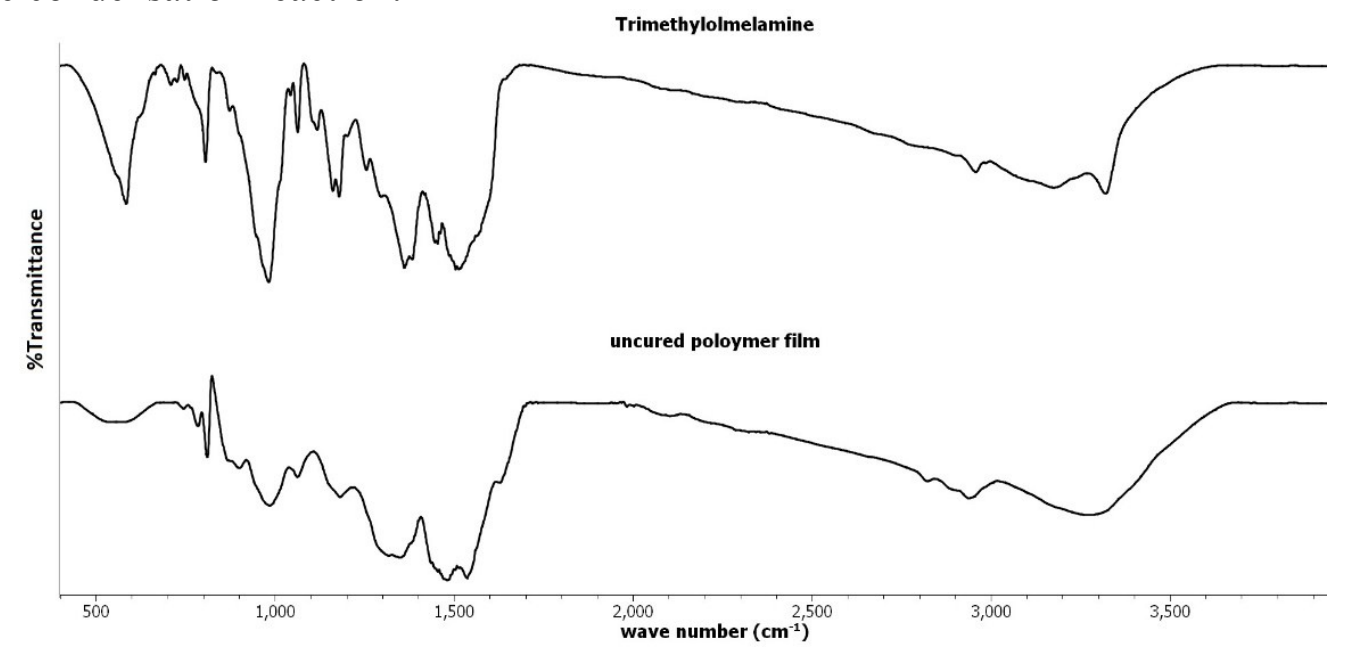

Fig. 2 The comparison of FTIR spectra of trimethylolmelamine (TMM) and uncured MF polymer film in gel state

Fig. 3 shows the FTIR spectra obtained from cured samples under different conditions compared to that of the uncured gel. The disappearance of the characteristic methylol C-H vibrations band can be observed in cured samples, which suggests that further condensation reaction occurs during the 
curing process. However there is no pronounced difference among cured samples at different temperature and time. This suggests that the complete condensation reaction could be possibly reached at relatively low curing temperature and short duration of time, e.g., at $100{ }^{\circ} \mathrm{C}$ for 1 hour.

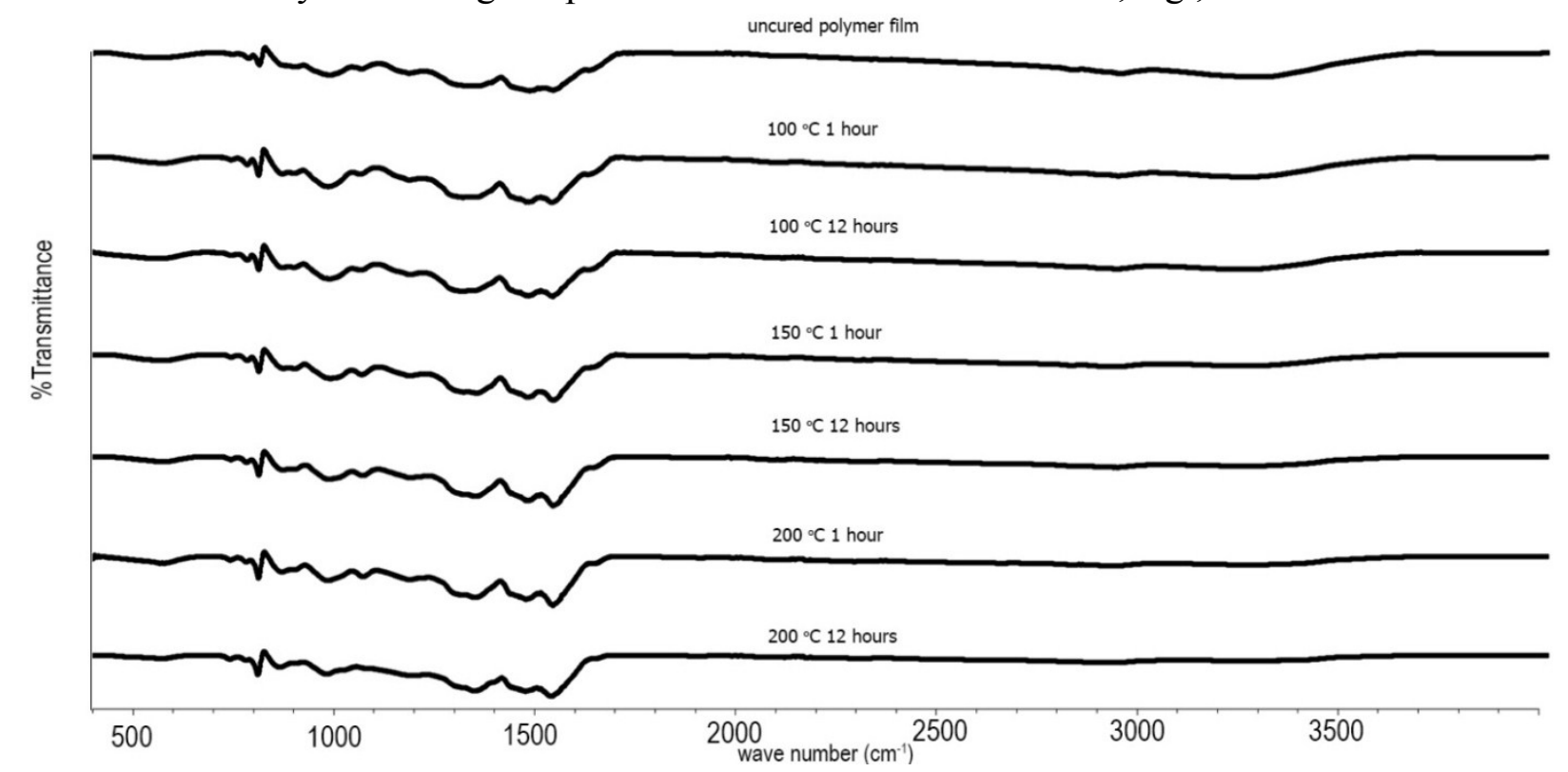

Fig. 3 The comparison of FTIR spectra of the uncured gel and the cured samples at different temperature and time durations

TGA curves obtained under normal atmosphere obtained from the uncured polymer film and all other samples cured under different condition show and interesting feature. Regardless of the curing time, the samples cured at $100-150{ }^{\circ} \mathrm{C}$ start to decompose at approximately $330{ }^{\circ} \mathrm{C}$, whereas the sample cured at $200{ }^{\circ} \mathrm{C}$ starts to decompose at approximately $350{ }^{\circ} \mathrm{C}$. This suggests that further condensation reactions also occurs even in solid state and further reinforce the structural stability of the polymer network. However, more investigation such as solid state ${ }^{13} \mathrm{C}$-NMR is needed in order to confirm this assumption. The curing experiment was not done at any temperature higher than 200 ${ }^{\circ} \mathrm{C}$ because the onset of the mass loss under normal atmosphere starts to be observable at that temperature. Fig. 4 shows the comparison of TGA curves obtained from polymer films cured at 100 and $200{ }^{\circ} \mathrm{C}$ for 12 hours. The one cured at $200{ }^{\circ} \mathrm{C}$ distinctively shows the higher decomposition temperature which suggests stronger polymer network due to higher degree of condensation reaction.

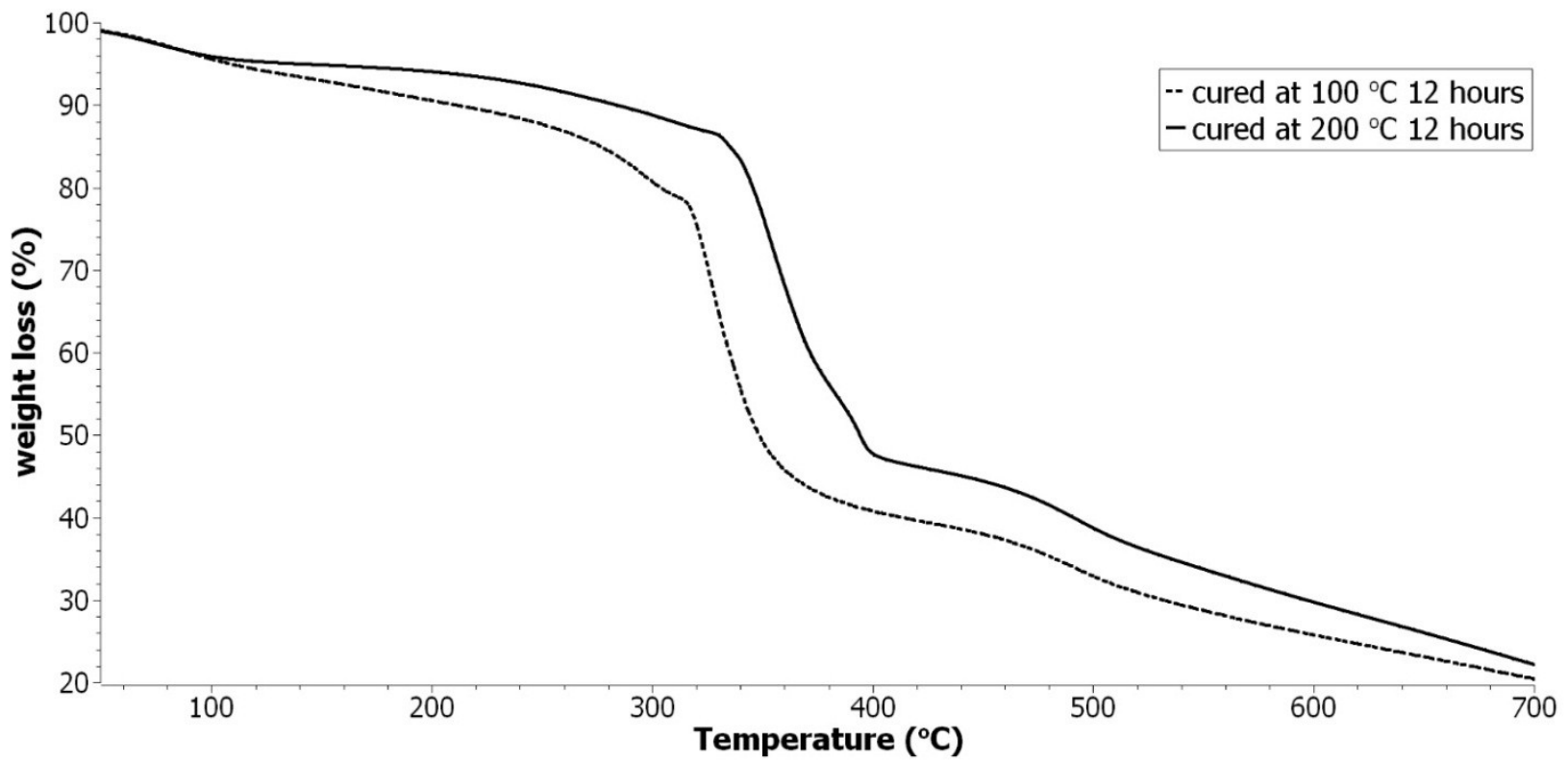

Fig. 4 TGA curves comparison of MF polymer films cured at 100 (dashed line) and $200{ }^{\circ} \mathrm{C}$ for 12 hours. 
To shed some light on to the curing mechanism and find the optimum curing temperature for and time MF polymer films in this experiment, DSC curves were obtained from the uncured sample and the samples cured at 100 and $200{ }^{\circ} \mathrm{C}$ for 12 hours respectively as shown in Fig 5. Some exothermic peaks can be observed from the uncured sample and the one cured at $100{ }^{\circ} \mathrm{C}$ even for 12 hours. However, no pronounced exothermic peak can be observed from the sample cured at $200{ }^{\circ} \mathrm{C}$ for the same length of time. This suggests that the polymer film cured at $200{ }^{\circ} \mathrm{C}$ has already undergone further condensation process and structural rearrangement more completely than the uncured film and the one cured at lower temperatures. The curing time also has some effect on the structure of the resulting polymer network as shown in Fig. 6. DSC curves of samples cured at $200{ }^{\circ} \mathrm{C}$ for different time durations show different features. No pronounced exothermic peaks can be observed from the sample cured for 12 hours. This suggests that the structural rearrangement process during the curing procedure could be thermodynamically controlled comparable to those, although in different systems, covalent triazine frameworks. [6,10]

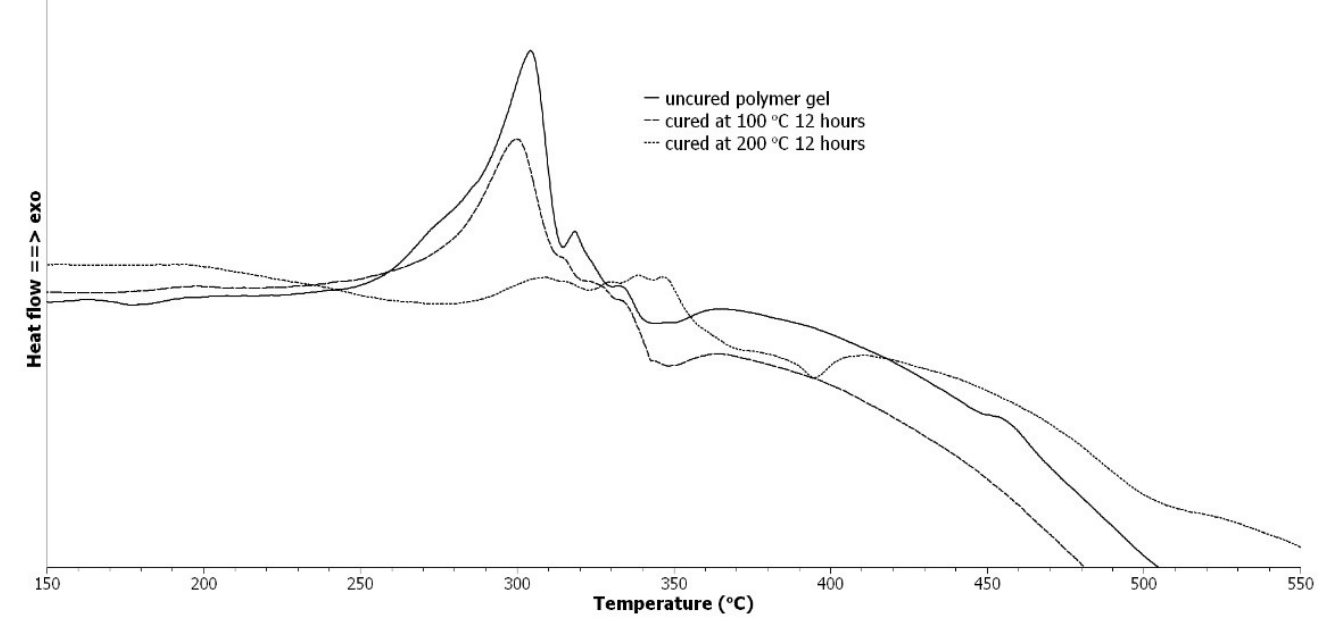

Fig. 5 DSC curves comparison between uncured and cured samples of MF polymer networks at 100 and $200{ }^{\circ} \mathrm{C}$ for 12 hours

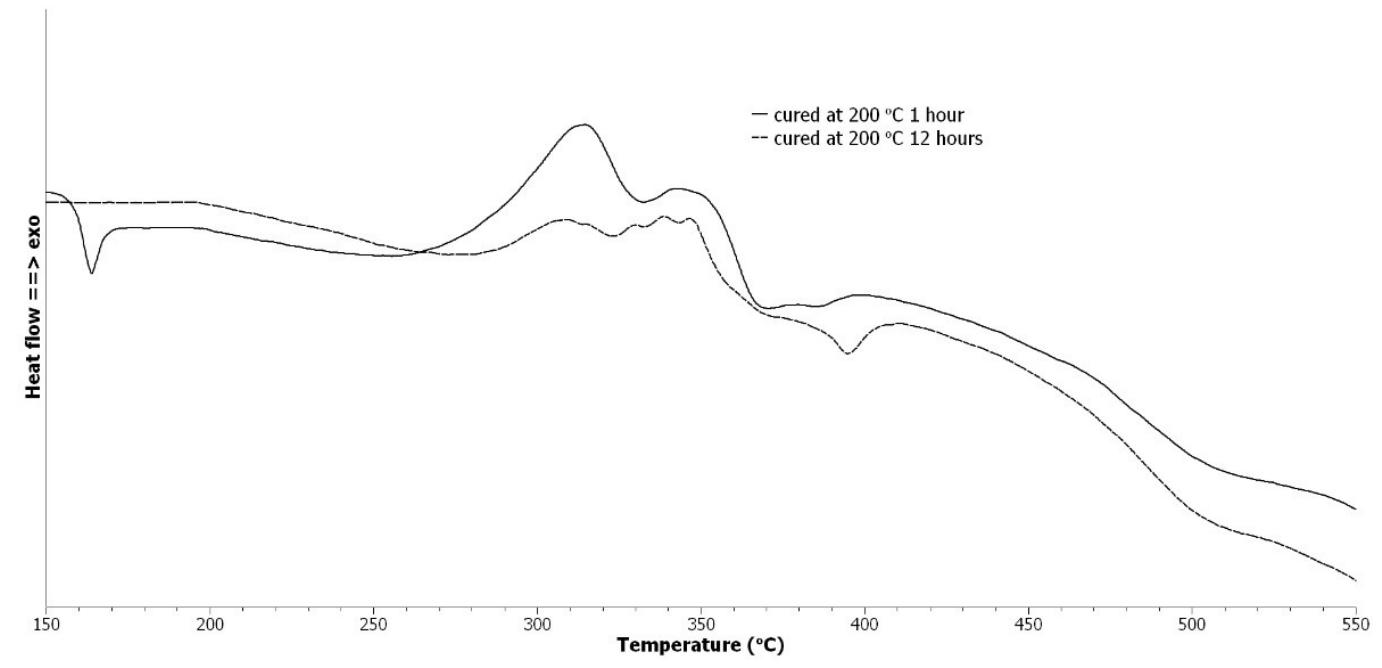

Fig. 6 DSC curves comparison between samples cured at $200{ }^{\circ} \mathrm{C}$ for 1 and 12 hours.

\section{Conclusion}

This work reveals a cheap and effective way to the synthesis of MF resin polymer films with self-supporting morphology similar to the ones synthesized from HMMM.[7] This work also concludes that some chemical processes such as further condensation and structural rearrangement are still going on at the temperature above $150{ }^{\circ} \mathrm{C}$ which is the typical curing temperature for such materials. The curing time is also important to the structural stability of the cured materials. Longer curing time is proven to be more effective towards obtaining material with higher structural stability 
as shown by thermal analysis. This work also paves a new route towards mesoporous melamine resins materials and even new advanced porous composite materials with cheaper and more accessible starting reagents.

\section{Acknowledgement}

The author would like to cordially thank the department of Chemistry, Naresuan University for all the help it provides. Special thanks to Dr. Duangdao Channei and Ms. Amornrat Muangon from the department of chemistry, Naresuan University and to Dr. Rapipan Janmanee from the department of chemistry, Pibulsongkram Rajabhat University for all their helps and encouragements.

\section{Reference}

[1] B. -D. Park, H. -W. Jeong, Cure kinetics of melamine-formaldehyde resin/clay/cellulose nanocomposites. J. Ind. Eng. Chem. (2010) 16, 375-379.

[2] K. R. Dipak, J. P. Amit, N. N. Bhavi, A Study on composites from casein modified melamine formaldehyde resin. Poly. Plast. Tech. Eng. 45 (2006) 293-299.

[3] K. Anil, K. Vimal, Modelling and experimental investigation of melamine formaldehyde polymerization. Macromolecules 23 (1990) 3729-3736.

[4] D. M. Snyder, T. J. J. Vuk, Appl. Polym. Sci. 46 (1992) 1301.

[5] C.E. Chan-thaw, A. Villa, P. Katekomol, D. Su, A. Thomas, L. Prati, Covalent triazine framework as catalytic support for liquid phase reaction. Nano Lett. 10 (2010) 537-541.

[6] P. Katekomol, J. Roeser, M. Bojdys, J. Weber, A. Thomas, Covalent Triazine Frameworks Prepared from 1,3,5-Tricyanobenzene. Chem. Mater. 25 (2013) 1542-1548.

[7] K. Kailasam, Y.-S. Jun, P. Katekomol, J. D. Epping, W. H. Hong, A. Thomas, Mesoporous melamine resins by soft templating of block-co-polymer mesophases. Chem. Mater. 22 (2010) $428-434$.

[8] D. J. Merline, S. Vukusic, A. A. Abdala, Melamine formaldehyde: curing studies and reaction mechanism. Polymer Journal. 45 (2013) 413-419.

[9] T.B. Shah, H.S. Patel, T.B. Mehta, Synthesis, characterization and glass-reinforced composits of trimethylolmelamine-phenol resins. Intern. J. Polymeric Mater. 49 (2001) 238-239.

[10] P. Kuhn, M. Antonietti, A. Thomas, Porous, Covalent Triazine-Based Frameworks Prepared by Ionothermal Synthesis. Angew. Chem. Int. Ed. 47 (2008) 3450-3453 